\title{
ALGEBRAIC MESH QUALITY METRICS
}

\author{
PATRICK M. KNUPP *†
}

\begin{abstract}
Quality metrics for structured and unstructured mesh generation are placed within an algebraic framework to form a mathematical theory of mesh quality metrics. The theory, based on the Jacobian and related matrices, provides a means of constructing, classifying, and evaluating mesh quality metrics. The Jacobian matrix is factored into geometrically meaningful parts. A nodallyinvariant Jacobian matrix can be defined for simplicial elements using a weight matrix derived from the Jacobian matrix of an ideal reference element. Scale and orientation-invariant algebraic mesh quality metrics are defined. The singular value decomposition is used to study relationships between metrics. Equivalence of the element condition number and mean ratio metrics is proved. Condition number is shown to measure the distance of an element to the set of degenerate elements. Algebraic measures for skew, length ratio, shape, volume, and orientation are defined abstractly, with specific examples given. Combined metrics for shape and volume, shape-volume-orientation are algebraically defined and examples of such metrics are given. Algebraic mesh quality metrics are extended to non-simplicial elements. A series of numerical tests verify the theoretical properties of the metrics defined.
\end{abstract}

Key words. unstructured mesh generation, mesh quality metrics, condition number, shape



AMS subject classification. $65 \mathrm{M} 50$

\section{Introduction}

Mesh quality metrics for assessing the results of a meshing process have been in use almost since the begining of meshing. Metrics are or can be used in a number of ways. First, metrics can serve as mesh requirement specifications prior to mesh creation. Element volume, shape, and orientation in various parts of the geometric domain can be specified in advance of meshing to enable the mesh generator to select proper algorithms and concentrate on the most difficult areas. Second, mesh improvement techniques such as smoothing, optimization, and edge swapping depend heavily on the use of quality metrics. Third, metrics often serve as a quality control mechanism. Given a mesh, is it of sufficent quality that it can be passed on to the consumer? Non-adaptive, $\grave{a}$ priori meshing of complex geometries is difficult, especially with nonsimplicial elements. As a result, mesh quality is not assured. Consumers of meshes for adaptive purposes should also be interested in quality metrics because $\mathrm{h}$-adaptive mesh refinement rarely will improve initial mesh quality. R-type adaptive procedures, in which mesh nodes are moved, can also make good use of mesh quality metrics. Given these uses, mesh quality metrics will be needed for the forseable future.

For the most part, mesh quality metrics are based on geometric criteria. For example, does a given element possess positive volume and a good shape? Element

\footnotetext{
* PARALLEL COMPUTING SCIENCES DEPARTMENT

SANDIA NATIONAL LABORATORIES

M/S 0847, P.O. BOX 5800

ALBUQUERQUE, NM 87185-0847

PKNUPP@SANDIA.GOV

$\dagger$ THIS WORK WAS FUNDED BY THE DEPARTMENT OF ENERGY'S

MATHEMATICS, INFORMATION AND COMPUTATIONAL SCIENCES PROGRAM

(SC-31) AND WAS PERFORMED AT SANDIA NATIONAL LABORATORIES.

SANDIA IS A MULTIPROGRAM LABORATORY OPERATED BY SANDIA

CORPORATION, A LOCKHEED MARTIN COMPANY, FOR THE UNITED

STATES DEPARTMENT OF ENERGY UNDER CONTRACT DE-ACO4-94AL85000.
} 


\section{DISCLAIMER}

This report was prepared as an account of work sponsored by an agency of the United States Government. Neither the United States Government nor any agency thereof, nor any of their employees, make any warranty, express or implied, or assumes any legal liability or responsibility for the accuracy, completeness, or usefulness of any information, apparatus, product, or process disclosed, or represents that its use would not infringe privately owned rights. Reference herein to any specific commercial product, process, or service by trade name, trademark, manufacturer, or otherwise does not necessarily constitute or imply its endorsement, recommendation, or favoring by the United States Government or any agency thereof. The views and opinions of authors expressed herein do not necessarily state or reflect those of the United States Government or any agency thereof. 


\section{DISCLAIMER}

Portions of this document may be illegible in electronic image products. Images are produced from the best available original document. 
volume, aspect ratio, skew, angles, stretching, and orientation are common geometric quality metrics. Suprisingly, a mathematical theory of geometric mesh qualitymetrics has not been developed until now. Such a theory should include a discussion of what a mesh quality metric is, what properties should it possess, a capability for analyzing and classifying various metrics, including a way to show how metrics are related and a means of identifying redundant metrics. This attempt at such a theory is based on element Jacobian matrices and an algebraic framework that uses matrix norm, trace, and determinant. A crucial feature introduced in this theory is the idea that metrics don't exist in a vacuum, but need to be referenced to an ideal element. The metric then measures the deviation from the ideal. The ideal may vary from one application to another. For example, some applications can do well with isotropic elements while others may need anisotropic elements with particular orientations. We thus construct our theory for arbitrary reference elements.

We do not attempt a comprehensive survey of all the work that has been done on metrics, but refer the reader to the early work of Robinson on quality metrics for quadrilaterals [20], [21], [22], the distortion measure of Oddy [18], the 'flatness' measure of Ives [10], the summary of tetrahedral measures in [6], [19], and the measures in Canann [3]. The work reported here is an extension of the ideas of the author presented in [12], [13], [7], [14], and [15] in which the use of matrices, norms, and the condition number for mesh quality measures were introduced.

\section{Preliminary Observations}

For both structured and unstructured meshes we can refer to mesh nodes and mesh elements. A mesh element is a geometric object topologically equivalent to some geometrically regular object such as a cube/square, tetrahedron/triangle, wedge, or pyramid. The boundary of the element is defined in terms of mesh nodes with given spatial coordinates. ${ }^{1}$ Given a mesh element we define an element quality metric as follows.

\section{Definition.}

An element quality metric is a scalar function of node positions that measures some geometric property of the element.

If a three-dimensional element has $\mathrm{K}$ nodes with coordinates $x_{k} \in R^{3}, k=0,1, \ldots, K-$ 1 , then we denote a mesh quality metric by $\hat{f} \mid R^{3 K} \rightarrow R$.

A host of mesh quality metrics have been defined over the years. Many of the metrics are redundant. Others may lack one or more of the following desirable properties of quality metrics:

\section{Definitions}

A metric is dimension-free if its definition in $3 \mathrm{D}$ is an unambiguous, natural generalization of its definition in 2D, otherwise it is dimension-specific. Example: Volume metrics are dimension-free while angle metrics are dimension-specific.

\footnotetext{
${ }^{1}$ In this paper attention is restricted to linear elements having no mid-side nodes.
} 
A metric is element-free if its definition on one element type is an unambiguous, natural generalization of its definition on another element type, otherwise it is element-specific. Example: Maximum angle is element-free on two-dimensional elements while the ratio of quadrilateral diagonal lengths is element-specific.

A metric on a fixed element type is domain-general if it is meaningful over a wide range of possible shapes of the element, otherwise it is domain-specific. Example: Aspect ratio is domain specific. Although aspect ratio may be defined for any quadrilateral [22] it is not meaningful for any shape of quadrilateral. The minimum angle of a quadrilateral is domain-general.

A metric on a fixed element type is versatile if it is sensitive to more than one distortion mode (e.g., skew and aspect ratio), otherwise it is specialized. Example: Tetrahedral shape measures are versatile while skew is specialized. Versatile metrics are useful when one does not need to know the specific mode of distortion.

A metric is scale-free if it's value does not depend on the volume of the element, otherwise it is scale-sensitive. A metric is orientation-free if it's value does not depend on the orientation of the element in space, otherwise it is orientationsensitive. Example: Rectangle aspect ratio is scale-free and orientation-free. Volume is orientation-free, but scale-sensitive.

A metric is unitless if it has no units. Example: aspect ratio and skew are unitless while volume is not. Unitless measures do not depend upon the physical units of the problem (such as length in feet vs. meters).

A metric is referenced if it incorporates a comparison to a reference element, which may determine volume, shape, or orientation, otherwise it is unreferenced. Example, aspect ratio $h /(s w)$ is referenced to a rectangle with aspect ratio $s>0$. By necessity, referenced metrics are unitless.

In this paper we formulate metrics having these desirable properties.

Before proceeding we make a few general comments. First, many of the Propositions noted have trivial proofs, which are omitted. Proofs are given for less straightforward results. Second, although many of the ideas presented in this paper can be generalized, we prefer to remain concrete since the meshing application demands it. Accordingly, we work over the field of real numbers, with objects in two or three dimensions $(n=2$ or $n=3)$. We will work primarily with simplicial elements in mind and concentrate on three dimensions since this is more difficult than two dimensions. Most results we present hold in both two and three dimensions, even though only one case or the other is presented. Differences are noted. Extension of our results to non-simplicial elements is given in section 12 . We rely heavily on results from linear algebra to develop the theory of metrics. It is important to keep in mind that our emphasis differs from that of numerical linear algebra. The matrices with which we work are $2 \times 2$ or $3 \times 3$, so efficiency of computation is not the main issue. Instead, the issue is to define algebraic metrics having the desired properties and to show how they are related. 
Various sets of matrices are used extensively in our presentation. Let $\mathcal{M}_{n}$ be the set of all $n \times n$ real matrices. Let $\mathcal{M}_{n}^{+}$be the set of all $n \times n$ real matrices with positive determinant. The boundary of this latter set is $\partial \mathcal{M}_{n}^{+}$, the set of all $n \times n$ singular matrices. Let $\mathcal{I}_{n}$ be the $n \times n$ identity matrix and $\mathcal{O}$ the $n \times n$ zero-matrix. Let $\mathcal{Z}(n)$ be the set of all matrices in $\mathcal{M}_{n}^{+}$whose determinant is unity. Let $\mathcal{S O}(n)$ be the set of all $n \times n$ orthogonal matrices with determinant 1 . Let $\mathcal{D}(n)$ be the set of all $n \times n$ non-singular diagonal matrices and $\mathcal{U}(n)$ the set of all $n \times n$ non-singular upper triangular matrices. Let $\mathcal{S R}(n)$ be the set of all $n \times n$ non-singular matrices of the form $\rho \Theta$ where $\rho>0$ and $\Theta \in \mathcal{S O}(n)$. Each of these sets forms one of the classical matrix groups. Recognition of these groups is important because the closure, identity, and inverse properties of these matrix groups are heavily relied on throughout this exposition.

Consider the affine map associated with a tetrahedron. Let $x_{k} \in R^{3}, k=0,1,2,3$ be the coordinates of the four vertices of the tetrahedron in physical space. Let $\xi_{k}$, with $0 \leq \xi_{k} \leq 1$ and $\xi_{0}+\xi_{1}+\xi_{2}+\xi_{3}=1$ be four logical space coordinates and define the mapping from logical space to physical space by

$$
x(\xi)=\sum \xi_{k} x_{k}
$$

with $x \in R^{3}$.

This can be explicitly written

$$
x=\left(1-\xi_{1}-\xi_{2}-\xi_{3}\right) x_{0}+\xi_{1} x_{1}+\xi_{2} x_{2}+\xi_{3} x_{3}
$$

giving

$$
x=A_{0} u_{0}+x_{0}
$$

with $x=(x, y, z)^{t}, u_{0}=\left(\xi_{1}, \xi_{2}, \xi_{3}\right)^{t}$, and

$$
A_{0}=\left(\begin{array}{ccc}
x_{1}-x_{0} & x_{2}-x_{0} & x_{3}-x_{0} \\
y_{1}-y_{0} & y_{2}-y_{0} & y_{3}-y_{0} \\
z_{1}-z_{0} & z_{2}-z_{0} & z_{3}-z_{0}
\end{array}\right)
$$

Written in this form, one sees that $x$ is an affine map which takes points $u_{0}$ in the right tetrahedron with node coordinates $(0,0,0),(1,0,0),(0,1,0)$, and $(0,0,1)$ to points in a tetrahedron in physical space with the four nodes $x_{k}$. The vector $x_{0}$ controls translation of the element while the matrix $A_{0}$ controls volume, shape, and orientation of the element. We refer to $A_{0}$ as the Jacobian matrix because the columns $x_{k}-x_{0}$ of the matrix is the Jacobian of the affine map with respect to the logical variables, i.e., $A_{i j}=d x_{i} / d \xi_{j}$. The Jacobian matrix has units of length and is, in general, nonsymmetric. The formulation above also applies to triangular elements on a surface, provided the surface has a well-defined normal at every point.

\section{The Jacobian Matrix}

\subsection{Geometric Significance}

The Jacobian matrix of an element is important because it is well-defined for both $n=2$ and $n=3$. Basing element metrics on the Jacobian matrix thus makes it easy 
to devise metrics that are dimension-free. Furthermore, the Jacobian matrix contains information relating to the volume, shape, and orientation of an element. This can be understood more clearly by performing the QR factorization of the Jacobian matrix. The factorization decomposes the Jacobian matrix into several matrices with clear geometric interpretations. These matrices will be used to build mesh quality metrics in sections $7,8,9,10$, and 12 .

Let $\mathrm{A}$ be the Jacobian matrix and $\lambda_{i j}=\left[A^{t} A\right]_{i j}$ be the elements of the "metric tensor." Let $\alpha=\operatorname{det}(A)$. It is assumed that $0<|A|<\infty$ and $\alpha \geq 0$. Elements with $\alpha<0$ are inverted and will not be considered.

Proposition 3.1

Let $\mathrm{A}$ be the $n=2$ or $n=3$ Jacobian matrix. Then one can decompose $\mathrm{A}$ as follows

$$
A=R U=\mu R S=\mu R Q D=R Q \Delta
$$

where

- $R \in \mathcal{S O}(n)$.

- $U=\mu S$ with $U \in \mathcal{U}(n)$ and $U_{i i}>0$,

- $\mu$ is a non-negative scalar,

- $S=Q D$ with $S \in \mathcal{U}(n)$, and $S_{11}=1$,

- $Q \in \mathcal{U}(n)$ and has unit column vectors, and

- $D, \Delta \in \mathcal{D}(n)$.

\section{Proof}

We explicitly construct the factorizations for $n=2$ and $n=3$, as they are needed for computation of the various metrics to be defined later. For $n=2$,

$$
\begin{gathered}
R=\frac{1}{\sqrt{\lambda_{11}}}\left(\begin{array}{cc}
A_{11} & -A_{21} \\
A_{21} & A_{11}
\end{array}\right) \\
U=\left(\begin{array}{cc}
\sqrt{\lambda_{11}} & \lambda_{12} / \sqrt{\lambda_{11}} \\
0 & \alpha / \sqrt{\lambda_{11}}
\end{array}\right) \\
\mu=\sqrt{\lambda_{11}} \\
S=\left(\begin{array}{cc}
1 & \lambda_{12} / \lambda_{11} \\
0 & \alpha / \lambda_{11}
\end{array}\right) \\
Q=\left(\begin{array}{cc}
1 & \lambda_{12} / \sqrt{\lambda_{11} \lambda_{22}} \\
0 & \alpha / \sqrt{\lambda_{11} \lambda_{22}}
\end{array}\right) \\
D=\left(\begin{array}{cc}
1 & 0 \\
0 & \sqrt{\lambda_{22} / \lambda_{11}}
\end{array}\right) \\
\Delta=\left(\begin{array}{cc}
\sqrt{\lambda_{11}} & 0 \\
0 & \sqrt{\lambda_{22}}
\end{array}\right)
\end{gathered}
$$


For $n=3$, let $x_{\xi_{k}}, k=1,2,3$ be the $\mathrm{k}$-th column vector of $\mathrm{A}$.

$$
\begin{aligned}
& R=\left(\frac{x_{\xi_{1}}}{\sqrt{\lambda_{11}}}, \frac{\lambda_{11} x_{\xi_{2}}-\lambda_{12} x_{\xi_{1}}}{\sqrt{\lambda_{11}}\left|x_{\xi_{1}} \times x_{\xi_{2}}\right|}, \frac{x_{\xi_{1}} \times x_{\xi_{2}}}{\left|x_{\xi_{1}} \times x_{\xi_{2}}\right|}\right) \\
& U=\left(\begin{array}{ccc}
\sqrt{\lambda_{11}} & \frac{\lambda_{12}}{\sqrt{\lambda_{11}}} & \frac{\lambda_{13}}{\sqrt{\lambda_{11}}} \\
0 & \frac{\left|x_{\xi_{1}} \times x_{\xi_{2}}\right|}{\sqrt{\lambda_{11}}} & \frac{\lambda_{11} \lambda_{23}-\lambda_{12} \lambda_{13}}{\sqrt{\lambda_{11}\left|x_{\xi_{1}} \times x_{\xi_{2}}\right|}} \\
0 & 0 & \frac{\alpha}{\left|x_{\xi_{1}} \times x_{\xi_{2}}\right|}
\end{array}\right) \\
& \mu=\sqrt{\lambda_{11}} \\
& S=\left(\begin{array}{ccc}
1 & \frac{\lambda_{12}}{\lambda_{11}} & \frac{\lambda_{13}}{\lambda_{11}} \\
0 & \frac{\left|x_{\xi_{1}} \times x_{\xi_{2}}\right|}{\lambda_{11}} & \frac{\lambda_{11} \lambda_{23}-\lambda_{12} \lambda_{13}}{\lambda_{11 \mid}\left|x_{\xi_{1}} \times x_{\xi_{2}}\right|} \\
0 & 0 & \frac{\alpha}{\sqrt{\lambda_{11}\left|x_{\xi_{1}} \times x_{\xi_{2}}\right|}}
\end{array}\right) \\
& Q=\left(\begin{array}{ccc}
1 & \frac{\lambda_{12}}{\sqrt{\lambda_{11} \lambda_{22}}} & \frac{\lambda_{13}}{\sqrt{\lambda_{11} \lambda_{33}}} \\
0 & \frac{\left|x_{1} \times x_{\xi_{2}}\right|}{\sqrt{\lambda_{11} \lambda_{22}}} & \frac{\lambda_{11} \lambda_{23}-\lambda \lambda_{23} \lambda_{13}}{\sqrt{\lambda_{11} \lambda_{33}\left|x_{\xi_{1}} \times x_{\xi_{2}}\right|}} \\
0 & 0 & \frac{\alpha}{\sqrt{\lambda_{33}\left|x_{\xi_{1}} \times x_{\xi_{2}}\right|}}
\end{array}\right) \\
& D=\operatorname{diag}\left(1, \frac{\sqrt{\lambda_{22}}}{\sqrt{\lambda_{11}}}, \frac{\sqrt{\lambda_{33}}}{\sqrt{\lambda_{11}}}\right) \\
& \Delta=\operatorname{diag}\left(\sqrt{\lambda_{11}}, \sqrt{\lambda_{22}}, \sqrt{\lambda_{33}}\right)
\end{aligned}
$$

$\S$

The orientation matrix $\mathrm{R}$ rotates the first column vector of $\mathrm{A}$ to the $\mathrm{x}$-axis (and, for $n=3$, rotates the second column vector to the $\mathrm{x}-\mathrm{y}$ plane). The volume matrix $\mathrm{U}$ contains volume and shape information about the element, but not orientation. The scale factor $\mu$ is the length of the first column vector in the Jacobian matrix. The shape matrix $\mathrm{S}$ contains length ratio and skew information. The length ratio matrix $\mathrm{D}$ gives the ratio of element edge lengths while the skew matrix $\mathrm{Q}$ contains information about the angles in the element. The matrices R, S, Q, and D have units of $(\text { lengt } h)^{0}$ while $\mathrm{U}, \Delta$, and $\mu$ have units of (length $)^{1}$.

Orientation, volume, shape, length ratio, and skew are a complete list of the element properties embodied in the Jacobian matrix. Other properties such as curvature or relationships between adjacent elements are not contained in this matrix.

\subsection{Multiple Jacobian Matrices}

To obtain the Jacobian matrix $A_{0}$ of the affine map in the previous section we replaced 
$\xi_{0}$ with $1-\xi_{1}-\xi_{2}-\xi_{3} . A_{0}$ is thus referenced to the node at $x_{0}$. One could just as well refer to any of the four nodes in the tetrahedron, giving four Jacobian matrices per tetrahedral element. Let $k=0,1,2,3$ and

$$
A_{k}=(-1)^{k}\left(\begin{array}{lll}
e_{k+1, k} & e_{k+2, k} & e_{k+3, k}
\end{array}\right) \text {. }
$$

be the $\mathrm{k}$-th Jacobian matrix, where $e_{k, \ell}=\mathrm{x}_{k}-\mathrm{x}_{\ell}$ with $k \neq \ell$ and $\ell=0,1,2,3$ (note that $e_{\ell, k}=-e_{k, \ell}$ ). Node $\mathrm{k}$ has three attached edge vectors, $e_{k+1, k}, e_{k+2, k}$, and $e_{k+3, k}$, where the indices are taken modulo four. The $(-1)^{k}$ factor ensures that $\alpha_{k}>0$ according to the right-hand-rule.

Definitions Let $x_{k}$ be the nodes of a simplicial element $\varepsilon_{n}$. Let the centroid of the element be

$$
x_{c}=\frac{1}{n+1} \sum_{k} x_{k}
$$

Element Translation. Let $\tilde{x}_{k}$ be the corresponding nodes of the element translated in space by a vector $\mathrm{b}$. Then $\tilde{x}_{k}=x_{k}+b$ and the centroid of the translated element, $\tilde{x}_{c}$, is $\tilde{x}_{c}=x_{c}+b$.

Element Scaling. Let $\tilde{x}_{k}$ be the corresponding nodes of an element uniformly scaled by $\rho>0$ about the centroid. Then $\tilde{x}_{k}=x_{c}+\rho\left(x_{k}-x_{c}\right)$ and the centroid is $\tilde{x}_{c}=x_{c}$. Element Rotation. Let $\tilde{x}_{k}$ be the corresponding nodes of the element rotated about its centroid. Then, if the rotation is given by $\Theta \in \mathcal{S O}(n), \tilde{x}_{k}=x_{c}+\Theta\left(x_{k}-x_{c}\right)$ and the centroid of the rotated element is $\tilde{x}_{c}=x_{c}$.

Element Scaling and Rotation. Let $\tilde{x}_{k}$ be the corresponding nodes of the scaled and rotated element. Then $\tilde{x}_{k}=x_{c}+B\left(x_{k}-x_{c}\right)$ where $B \in \mathcal{S R}(n)$ and the centroid is preserved.

\section{Proposition 3.2}

The Jacobian matrices $A_{k}$ transform under element translation, scaling, rotation, or both scaling and rotation as $\tilde{A}_{k}=A_{k}$ (translation), $\tilde{A}_{k}=\rho A_{k}$ (uniform scaling), $\tilde{A}_{k}=\Theta A_{k}$ (rotation), and $\tilde{A}_{k}=B A_{k}$ (scaling and rotation). $\S$

\section{Proposition 3.3.}

If $A_{k}$ is given, the nodal coordinates are known up to a translation of $\varepsilon_{n}$.

The fact that $A_{k}$ is not invariant to $k$ would appear to be a serious obstacle to using the Jacobian matrix as a basis for measuring element quality because metrics based on $A_{k}$ will vary with $k^{2}$ This difficulty will be addressed in the next subsection but first we show how the four Jacobian matrices are related.

Let $M \in \mathcal{Z}(n)$ be the following constant matrix

$$
M=\left(\begin{array}{ccc}
1 & 1 & 1 \\
-1 & 0 & 0 \\
0 & -1 & .0
\end{array}\right)
$$

\section{Proposition 3.4.}

The set $\mathcal{I}_{3}, M, M^{2}, M^{3}$ is a cyclic group under matrix multiplication. $\S$

\footnotetext{
2 The matrices $\mathrm{R}, \mathrm{U}, \mathrm{S}, \mathrm{Q}, \mathrm{D}$, and $\Delta$ in the factorization of $\mathrm{A}$ are also not invariant to $k$.
} 
Proposition 3.5. The four Jacobian matrices are related to one another by $A_{k}=$ $A_{0} M^{k}$. This can be verified by a direct calculation. ${ }^{3} \S$

Proposition 3.6. The Jacobian determinant $\alpha_{k}$ is invariant to $\mathrm{k}$.

Proof. This follows directly from Proposition 3.5 since the determinant of $\mathrm{M}$ equals 1. $\S$

The result in Proposition 3.6 is to be expected since the volume of a tetrahedron is one-sixth of the Jacobian determinant [8], and hence $\alpha_{k}$ cannot depend on $\mathrm{k}$.

\subsection{A Nodally-invariant Jacobian Matrix}

In this section we exhibit a weighted Jacobian matrix that is nodally-invariant. We consider linear transformations between certain simplicial elements. The figure below illustrates the situation in two-dimensions. Three triangular elements are shown in the figure, the logical triangle, the reference triangle, and the physical triangle. The physical triangle is the triangle defined by an element of the mesh. The reference triangle is the ideal triangle one wants to obtain (for example, an equilateral triangle). The logical triangle is constructed by placing one node at the origin and the other nodes at unit lengths along Cartesian axes. The physical triangle has three Jacobian matrices $A_{k}$, defined in the previous section. Similarly, the reference triangle has three Jacobian matrices $W_{k}$, computed in the same manner. The logical triangle also has three Jacobian matrices, $\mathcal{I}_{n}, M$, and $M^{2}$, corresponding to $k=0,1,2$. The three triangles can be related via the three matrices $W_{k}, T_{k}=A_{k} W_{k}^{-1}$, and $A_{k}$. The matrix $W_{k}$ is taken to have the same units as $A_{k}$ (length), therefore $T_{k}$ is unitless.

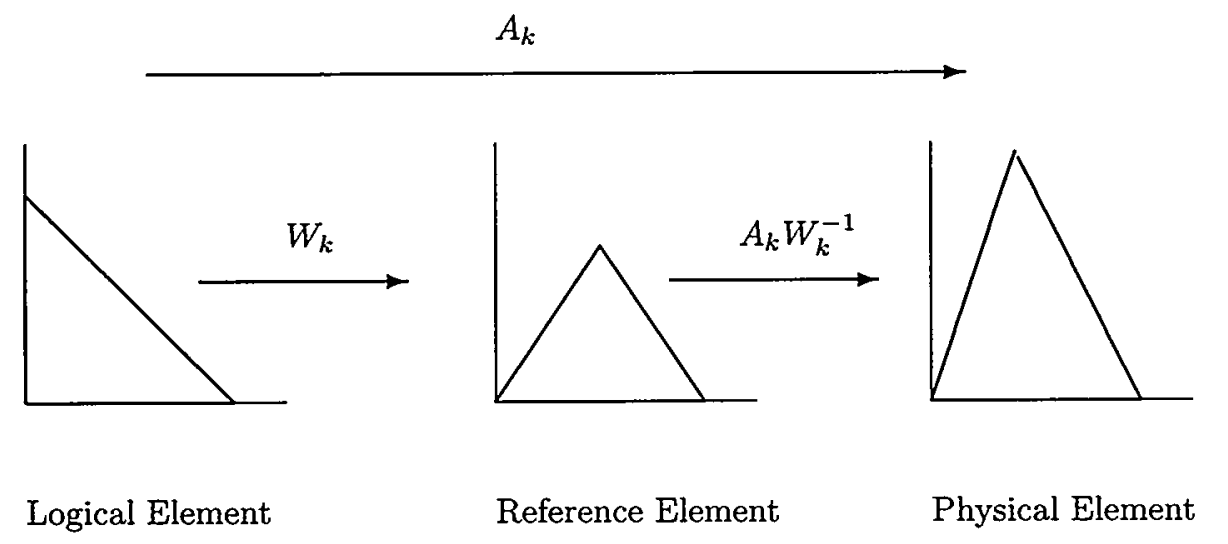

\section{Proposition 3.7.}

Given any tetrahedron with Jacobian matrices $A_{k}, k=0,1,2,3$, let $T_{k}$ be the linear transformation that takes $W_{k}$ to $A_{k}$. Assume $\operatorname{det}\left(W_{k}\right) \neq 0$. Then $T_{k}=A_{0} W_{0}^{-1}$, that is, $T_{k}$ is independent of $k$.

\footnotetext{
${ }^{3}$ For $n=2$,
}

$$
M=\left(\begin{array}{cc}
-1 & -1 \\
1 & 0
\end{array}\right)
$$




\section{Proof.}

By definition, $T_{k} W_{k}=A_{k}$. Proposition 3.5 applies to the matrices $W_{k}$. Thus $W_{k}=W_{0} M^{k}$. Since $A_{k}=A_{0} M^{k}$, we have the stated result. $\S$

The matrix $T=A W^{-1}$ between the reference and physical elements does not depend on which node one chooses to compute, therefore one may use $T$ (instead of A) to define nodally-invariant element quality measures.

A consequence of the nodal invariance of $T$ is that, unlike geometrically-based tetrahedral metrics [19], we do not use all the edges of the tetrahedron, but only three (however, we also use three edges of the reference element).

From here on, then, we suppress the subscripts $k$, with the understanding that $A$ and $\mathrm{W}$ must be computed with respect to the same node. This implies a one-to-one correspondance between the nodes of the reference element and the physical element. The matrix $\mathrm{W}$ is not only useful for making $\mathrm{T}$ nodally-invariant but, as will be seen, it permits the construction of referenced quality metrics. Because $\mathrm{W}$ is derived from an ideal reference element, it is reasonable to assume that $w=\operatorname{det}(W)>0$.

The following associated derived matrices are useful in a theory of quality metrics: $T^{t}, T^{-1}, T^{-t}, \operatorname{adj}(T), T^{t} T$ (the metric matrix), $\left(T^{t} T\right)^{-1}$, and $T T^{t} T$.

\section{Algebraic Mesh Quality Meausures}

We have shown that, given $\mathrm{W}$, the nodally-invariant Jacobian matrix $\mathrm{T}$ can be computed using any node of a simplicial element. The Jacobian matrix A was factored into four matrices controlling orientation, volume, skew, and length ratio. We now turn to the question of how to build mesh quality metrics from these matrices. Determinant, trace, and norm are the most useful means to convert matrices to scalar quantities.

Definition. Let $\tau=\operatorname{det}(T)=\operatorname{det}\left(A W^{-1}\right)=\operatorname{det}(A) \operatorname{det}\left(W^{-1}\right)=\alpha / w$.

\section{Proposition 4.1.}

$\alpha$ and $\tau$ are invariant to element rotation because $\operatorname{det}(\Theta T)=\operatorname{det}(T)$.

Another useful means to convert a matrix to a scalar is the trace function.

\section{Definition.}

$$
\operatorname{trace}(T)=\sum_{i} T_{i i}
$$

\section{Proposition 4.2.}

$\operatorname{trace}(T)$ is a linear map from $\mathcal{M}_{n}$ to the real numbers, i.e., $\operatorname{trace}(\rho T)=\rho \operatorname{trace}(T)$ and $\operatorname{trace}\left(T_{1}+T_{2}\right)=\operatorname{trace}\left(T_{1}\right)+\operatorname{trace}\left(T_{2}\right) . \S$

The matrix inner product $B \cdot C$, defined in terms of the trace, is trace $\left(B^{t} C\right)$. For example, $A^{t} \cdot W^{-1}=\operatorname{trace}(T)$. The inner product leads to the Frobenius matrix norm

$$
|T|^{2}=\operatorname{trace}\left(T^{t} T\right)
$$


The Frobenius norm is the sum of the squares of the matrix elements. The Frobenius norm is preferred for mesh quality metrics because (1) it is less expensive to compute than the p-norms and (2) many well-known mesh quality measures can be written in terms of the Frobenius norm. For some of the results in this paper it is necessary to use the 2-norm, which we will denote by $|T|_{2}$. The 2-norm of $T$ is the square-root of the maximum eigenvalue of $T^{t} T$.

Definition. Let $f \mid B_{i} \in \mathcal{M}_{n}, i=0, \ldots, I \rightarrow R$ be a continuous function from sets of real matrices to the real numbers. Then $f$ is an algebraic mesh quality metric if (1) the matrices $B_{i}$ are constructed from $A_{k}, W_{k}$ or factorizations thereof, (2) the matrices $B_{i}$ are converted to scalars by means of the matrix norm, determinant, or trace, and (3) $f$ is invariant to the element node at which the matrices are computed. The algebraic metric $f$ is referenced if the domain of $f$ is restricted to weighted matrices that make use of $\mathrm{W}$ or factorizations thereof.

Let $\mathcal{A}$ be the set of all algebraic mesh quality metrics.

TABLE 1

Examples of Algebraic Mesh Quality Metrics

\begin{tabular}{|c|c|}
\hline Algebraic Metric & Comments/Source \\
\hline trace $(T)$ & \\
\hline$T \cdot B$ & with B some constant matrix \\
\hline$|T|^{2}$ & Laplace \\
\hline$\left|T^{t} T\right|^{2}$ & Liao [16] \\
\hline$\tau^{2}$ & Volume [4] \\
\hline $\mid$ adj(T) $\left.\right|^{2}$ & Jacquotte [1]] \\
\hline$\tau\left|T^{-1}\right|^{2}$ & Winslow [2] \\
\hline$|T|^{2} / \tau$ & Barerra [1] \\
\hline$\tau^{-4 / 3}\left\{\left|T^{t} T\right|^{2}-(1 / 3)|T|^{4}\right\}$ & Oddy [18] \\
\hline$\tau^{2 / 3}\left|T^{-1}\right|^{2}$ & Non-dimensional Winslow \\
\hline$\tau^{-2 / 3}|T|^{2}$ & Mean Ratio ${ }^{-1}[17]$ \\
\hline$|T|\left|T^{-1}\right|$ & Condition Number [15] \\
\hline
\end{tabular}

Examples of algebraic metrics are given in Table 1. They are inspired by the sources cited but these sources did not pose the metrics in terms of the Jacobian matrix. Furthermore, none of the metrics in the sources were explicitly referenced.

Algebraic mesh quality metrics are, in general, no more expensive to compute than geometrically-based metrics, especially if the Frobenius norm is used.

An advantage of the algebraic metrics is that, using matix theory and linear algebra, they are in general easier to analyze than are non-algebraic metrics.

\section{Proposition 4.3}

Assume $\operatorname{det}(W)$ and $|W|>0$. Then (1) $\tau=0$ if and only if $\alpha=0,(2) \tau>0$ if and only if $\alpha>0$, (3) $|T|=0$ if and only if $|A|=0$, and (4) $|T|>0$ if and only if $|A|>0 . \S$

Let $f\left(\left\{B_{i}\right\}\right)$ be shorthand for $f\left(B_{1}, B_{2}, \ldots, B_{I}\right)$.

Definition. $f$ is scale-invariant if $f\left(\left\{\rho B_{i}\right\}\right)=f\left(\left\{B_{i}\right\}\right)$ for $\rho>0$. 
Example: For $n=3, \tau^{-2 / 3}|T|^{2}$ is scale invariant, while for $n=2$ it is not.

Let $\Theta \in \mathcal{S O}(n)$. From the defnintion of the Frobenius norm it is easy to show that $|T \Theta|=|T|$ and $|\Theta T|=|T|$, i.e., the Frobenius norm is invariant to rotations of the element. Because of this property, many natural algebraic metrics are orientation-free.

Definition. Let $f$ be an algebraic metric. Then $f$ is orientation-invariant if $f\left(\left\{\Theta_{1} B_{i} \Theta_{2}\right\}\right)=$ $f\left(\left\{B_{i}\right\}\right)$ for $\Theta_{1}, \Theta_{2} \in \mathcal{S O}(n){ }^{4}$

Examples:

$$
\begin{gathered}
f(T)=|T| \\
f(T)=\operatorname{det}(T)
\end{gathered}
$$

Definition. $f$ is scale and orientation-invariant if $f\left(\left\{H_{1} B_{2} H_{2}\right\}\right)=f\left(\left\{B_{2}\right\}\right)$ where $H_{1}, H_{2} \in \mathcal{S R}(n)$.

Example: $\kappa(T)=|T|\left|T^{-1}\right|$.

Definition. $f$ is positive if $f\left(\left\{B_{i}\right\}\right)>0$ for all $B_{i} \neq \mathcal{O}$.

Example: $f(T)=|T|$.

Definition. $f$ is even if $f\left(\left\{-B_{i}\right\}\right)=f\left(\left\{B_{i}\right\}\right) . \mathrm{f}$ is odd if $f\left(\left\{-B_{i}\right\}\right)=-f\left(\left\{B_{i}\right\}\right)$.

Example: $\operatorname{trace}(T)$ is odd, $|T|$ is even, and $\operatorname{det}(T)$ is odd when $\mathrm{n}$ is odd and even when $\mathrm{n}$ is even.

Definition. $f$ is Transpose Invariant if $f\left(\left\{B_{i}^{t}\right\}\right)=f\left(\left\{B_{i}\right\}\right)$.

Example: $f(T)=|T|$.

Since norm, determinant, and trace are all invariant to matrix transpose, the majority of mesh quality metrics are transpose invariant. An example of a metric that is not transpose invariant is $f(T)=|T-C|$ where $\mathrm{C}$ is an arbitrary constant matrix.

Definition. The conjugate metric of $f\left(\left\{B_{i}\right\}\right)$ is $f^{*}\left(\left\{B_{i}\right\}\right)=f\left(\left\{B_{i}^{-t}\right\}\right)$. Note that $f^{* *}=f$.

Example: For $n=3, \mu(T)=\tau^{-2 / 3}|T|^{2}$ has conjugate $\mu^{*}(T)=\tau^{2 / 3}\left|T^{-1}\right|^{2}$. Thus the mean ratio metric is conjugate to the modified Winslow metric.

Definition. $f$ is self-conjugate if $f^{*}=f$.

Examples:

$$
\begin{array}{r}
f(T)=\kappa(T)=|T|\left|T^{-1}\right| \\
f(T)=\tau^{-2 / 3}|T|^{2}+\tau^{2 / 3}\left|T^{-1}\right|^{2}
\end{array}
$$

\footnotetext{
${ }^{4} f$ is left orientation-invariant if $f\left(\left\{\Theta_{1} B_{i}\right\}=f\left(\left\{B_{i}\right\}\right)\right.$. Example: $f(T)=\left|T^{t} T-I\right|$.
} 


\section{Singular Values}

In this section we show that algebraic mesh quality metrics may be expressed in terms of singular values and that this provides a useful tool in analyzing properties of such metrics.

The singular value decomposition of a matrix $T$ says there exists $\Theta, \Phi \in \mathcal{S O}(n)$ such that

$$
T=\Theta^{t} D \Phi
$$

where $D=\operatorname{diag}\left(\sigma_{1}, \sigma_{2}, \sigma_{3}\right) \in \mathcal{D}(n)$. The singular values $\sigma_{k}(T), k=1,2,3$, are real and positive. They are related to the eigenvalues of $T^{t} T$ by $\sigma_{k}(T)=\sqrt{\lambda_{k}\left(T^{t} T\right)}$. Hence $|T|_{2}=\sqrt{\lambda_{\max }}=\sigma_{\max }$.

Let $\sigma(T)=\left(\sigma_{1}, \sigma_{2}, \sigma_{3}\right)^{t} \in R^{3}$ be the vector of singular values of $\mathrm{T}$. Let $\lambda\left(T^{t} T\right)=$ $\left(\lambda_{1}, \lambda_{2}, \lambda_{3}\right)^{t} \in R^{3}$ be the vector of eigenvalues of $T^{t} T$. Then $\sigma(T)$ and $\lambda(T)$ map $T \in \mathcal{M}_{n}$ to vectors in $R^{3}$.

\section{Proposition 5.1}

For $\tau>0, \Psi \in S O(n)$, and $\rho>0$,

$$
\begin{aligned}
\sigma\left(T^{t}\right) & =\sigma(T) \\
\sigma(\Psi T) & =\sigma(T) \\
\sigma(\rho T) & =\rho \sigma(T) \\
\sigma_{k}\left(T^{-1}\right) & =1 / \sigma_{k}(T) \\
\sigma_{k}\left(T^{t} T\right) & =\sigma_{k}^{2}(T)
\end{aligned}
$$

$\S$

\section{Proposition 5.2}

$$
\begin{aligned}
|T|^{2} & =\sum_{k} \sigma_{k}^{2}(T)=\sum_{k} \lambda_{k}\left(T^{t} T\right) \\
\tau & =\prod_{k} \sigma_{k}(T)=\sqrt{\prod_{k} \lambda_{k}\left(T^{t} T\right)}
\end{aligned}
$$

Thus $\tau=0$ if and only if $\sigma_{\min }=0 . \S$

One can express algebraic metrics $f(T)$ as functions of the singular values $\tilde{f}(\sigma(T))$. Note that $\tilde{f}(\sigma)$ maps a vector in $R^{3}$ to a scalar. Thus $f=\tilde{f} \circ \sigma$ maps $T$ to a scalar, i.e., it is the composition of the two maps. For example, if $\tilde{f}(\sigma)=|\sigma|^{2}$, then $f(T)=|T|^{2}$. Some other examples are

$$
\begin{aligned}
\left|T^{t} T\right|^{2} & =\sum_{k} \sigma_{k}^{4}=\sum_{k} \lambda_{k}^{2} \\
|T|^{4} & =|\sigma|^{4}=\left(\sum_{k} \lambda_{k}\right)^{2}
\end{aligned}
$$




$$
\begin{aligned}
|\operatorname{adj} T|^{2} & =\sum_{l} \prod_{k \neq \ell} \sigma_{k}^{2}=\sum_{\ell} \prod_{k \neq \ell} \lambda_{k} \\
\left|T^{-1}\right|^{2} & =\sum_{k}\left(1 / \sigma_{k}\right)^{2}=\sum_{k} 1 / \lambda_{k}
\end{aligned}
$$

Given $f(T)$, the corresponding function $\tilde{f}(\sigma)$ always exists because the SVD of $\mathrm{T}$ always exists. Thus, algebraic mesh quality metrics may always be expressed in terms of singular values. On the other hand, given some arbitrary function $\tilde{f}$ of $\sigma$, there may not correspond an algebraic mesh quality metric $f(T)$. For example: $\tilde{f}(\sigma)=\sum_{k} \sin \sigma_{k}$ cannot be derived from a mesh quality metric.

\section{Proposition 5.3}

$f(T)=\operatorname{trace}(T)$ gives rise to a linear function $\tilde{f}$ of its singular values of the form $\tilde{f}(\sigma)=t \cdot \sigma$. The vector $t$ has components $t_{\ell}=\sum_{k} \Theta_{\ell, k} \Phi_{\ell, k}$.

Definition. $f$ is homogeneous of degree $m$ if for $\rho>0, f\left(\left\{\rho B_{i}\right\}\right)=\rho^{m} f\left(\left\{B_{i}\right\}\right)$. Metrics with no such property are inhomogeneous.

Examples: $f(T)=\tau$ is homogeneous of degree $n . f(T)=\operatorname{trace}(T)$ and $|T|$ are homogeneous of degree $1 .|T|^{2}+\tau^{2}$ is inhomogeneous for both $n=2,3$.

\section{Proposition 5.4}

Let $f(T)$ be homogeneous of degree $\mathrm{m}$. Then the product $f^{*} f$ is homogeneous of degree 0 , i.e., scale-invariant. $\S$

Example: $f(T)=|T|^{2}$ gives $\left(f^{*} f\right)(T)=\kappa^{2}(T)$.

Definition Let $\mathcal{A}_{H}^{m} \subset \mathcal{A}$ be the set of all homogeneous algebraic metrics of degree $\mathrm{m}$.

\section{Proposition 5.5}

Let $f_{1} \in \mathcal{A}_{H}^{m}$ and $f_{2} \in \mathcal{A}_{H}^{\ell}$. Then $f_{1} f_{2} \in \mathcal{A}_{H}^{m+\ell}$. $\S$

From this we observe that we can generate metrics having any degree of homogeneity.

\section{Proposition 5.6}

Let $f_{1} \in \mathcal{A}_{H}^{m}$ and $f_{2} \in \mathcal{A}_{H}^{m}$. Then $f_{1}+f_{2} \in \mathcal{A}_{H}^{m}$. $\S$

Definition. Let $\tilde{\mathcal{A}}$ be the set of functions $\tilde{f}(\sigma)$ derived from the set $\mathcal{A}$ of algebraic mesh quality metrics. Let $\tilde{\mathcal{A}}_{H}^{m} \subset \tilde{\mathcal{A}}$ be the set of functions in $\tilde{\mathcal{A}}$ that are homogeneous of degree $\mathrm{m}$.

Proposition 5.7

If $f \in \mathcal{A}_{H}^{m}$, then $\tilde{f} \in \tilde{\mathcal{A}}_{H}^{m}$.

Proof

If $f\left(\left\{\rho B_{i}\right\}\right)=\rho^{m} f\left(\left\{B_{i}\right\}\right)$, then by definition,

$$
\tilde{f}\left(\sigma\left(\left\{\rho B_{i}\right\}\right)\right)=\rho^{m} \tilde{f}\left(\sigma\left(\left\{B_{i}\right\}\right)\right)
$$

But Proposition 5.1 then implies

$$
\tilde{f}(\rho \sigma)=\rho^{m} \tilde{f}(\sigma)
$$




\section{Proposition 5.8}

If $f$ is positive, so is $\tilde{f}$. If $f$ is even/odd, so is $\tilde{f}$. If $f$ is self-conjugate, so is $\tilde{f}$. $\S$

Singular values can be used to prove two important identities which hold for Frobenius norms of $3 \times 3$ matrices $^{5}$ :

\section{Proposition 5.9}

$$
\begin{array}{r}
\left|T^{t} T\right|^{2}+2 \tau^{2}\left|T^{-1}\right|^{2} \equiv|T|^{4} \\
3|T|^{2}\left|T^{t} T\right|^{2}-|T|^{6}+6 \tau^{2} \equiv 2\left|T T^{t} T\right|^{2}
\end{array}
$$

$\S$

These identities give the following bounds for $T_{3 \times 3}$ :

$$
\begin{array}{r}
|\operatorname{adj} T| \leq\left|T^{t} T\right| \leq|T|^{2} \\
\left|T T^{t} T\right|^{2}-\frac{1}{2}|T|^{6} \leq 3 \tau^{2} \leq\left|T T^{t} T\right|^{2}+\frac{1}{2}|T|^{6}
\end{array}
$$

One can also relate $\kappa\left(T^{t} T\right)$ to $\kappa(T)$ using singular values:

$$
\kappa^{2}\left(T^{t} T\right) \equiv \kappa^{4}(T)+4 \kappa^{2}(T)-2\left(\tau^{-2}|T|^{6}+\tau^{2}\left|T^{-1}\right|^{6}\right)
$$

Singular values have an important application in analyzing the equivalence of certain quality metrics.

\section{Shape Measures and Equivalences}

Tetrahedral shape measures for detecting distorted elements abound in the literature [6]. The list of measures includes such well-known quantities such as the radius ratio [8], mean ratio [17], solid angle, and several aspect ratios [19].

Tetrahedral shape measures are formally defined in [6]

"... a continuous function that evaluates the quality of a tetrahedron. It must be invariant under translation, rotation, reflection, and uniform scaling of the tetrahedron. It must be maximum for the regular tetrahedron and it must be minimum for a degenerate tetrahedron. There is no local maximum other than the global maximum for a regular tetrahedron and there is no local minimum other than the global minimum for a degenerate tetrahedron. For the ease of comparison, it should be scaled to the interval $[0,1]$, and be 1 for the

5 The corresponding identities for $T_{2 \times 2}$ are

$$
\begin{array}{r}
|T|^{2} \equiv \frac{1}{2}\left|T-T^{t}\right|^{2}+\operatorname{trace}\left(T^{2}\right) \\
|T|^{4} \equiv\left|T^{t} T\right|^{2}+2 \tau^{2}
\end{array}
$$


regular tetrahedron and 0 for a degenerate tetrahedron."

This definition was used to show mean ratio and radius ratio are shape measures while minimum dihedral angle and edge ratio are not [6] .

Shape measures are clearly mesh quality metrics but, in general, they are not algebraic mesh quality metrics. One exception is the mean ratio shape measure $\eta$, whose definition is given in [17]

$$
\eta(T)=\frac{3 \tau^{2 / 3}}{|T|^{2}}
$$

Definition. Let $\mathrm{a}, \mathrm{b}, \mathrm{c}$ be elements in a set. Recall that an equivalence relation $\sim$ on this set holds if

- $a \sim a$ for any $a$,

- $a \sim b$ if $b \sim a$,

- $a \sim b$ and $b \sim c$ implies $a \sim c$.

Definition (Liu and Joe, 1994): Let $M_{1}$ and $M_{2}$ be tetrahedral shape measures. Then $M_{1} \sim M_{2}$ if there exist constants $0<c_{1} \leq c_{2}$ and $0<p \leq q$ such that

$$
c_{1} M_{1}^{p} \leq M_{2} \leq c_{2} M_{1}^{q}
$$

The equivalence is strong if $p=q$. We use the notation $M_{1} \simeq M_{2}$ for strong equivalence.

Informally, equivalent shape metrics sense the same shape distortions, grow large together, and grow small together. The original motivation for introducing the idea of equivalences was to reduce the list of shape measures to some manageable number. For example, the shape measures radius ratio, mean ratio, and sine of solid angle are equivalent [17].

Definition. The definion of shape measure equivalence can be generalized to include all positive algebraic mesh quality measures. The definition for the latter is the same as the former, except replace the phrase 'tetrahedral shape measures' with 'positive algebraic mesh quality measures.'

\section{Proposition 6.1}

$|T|^{2} \simeq\left|T^{t} T\right|$ and neither is a shape measure.

Proof. From a well-known equivalence that can be found in [9]

$$
|T|_{2} \leq|T| \leq \sqrt{n}|T|_{2}
$$

we have

$$
\left|T^{t} T\right|_{2} \leq\left|T^{t} T\right| \leq \sqrt{n}\left|T^{t} T\right|_{2}
$$

that is,

$$
\sigma_{\max }^{2}(T) \leq\left|T^{t} T\right| \leq \sqrt{n} \sigma_{\max }^{2}(T)
$$

From Proposition 5.2 one can show

$$
\sigma_{\max }^{2}(T) \leq|T|^{2} \leq n \sigma_{\max }^{2}(T)
$$


thus,

$$
\frac{1}{n}|T|^{2} \leq\left|T^{t} T\right| \leq \sqrt{n}|T|^{2}
$$

$\S$

Proposition 6.2

Let $\nu>0$ be given and $\mathrm{M}$ be an algebraic mesh quality measure. Then $M^{\nu} \simeq M . \S$ Strong equivalence thus does not force homogeneous metrics to have the same degree of homogeneity.

Proposition 6.3

Let $\nu>0$. Then $M_{1} \sim M_{2}$ if and only if $M_{1}^{\mu} \sim M_{2}$.

The statement that if two metrics are equivalent, then it does not matter which one is used is an over-exaggeration. For example,

$$
\kappa(A) / \kappa(W) \leq \kappa(T) \leq \kappa(A) \kappa(W)
$$

shows the strong equivalence of $\kappa(A)$ and $\kappa(T)$, yet the weight matrix W is a critical factor in assessing the quality of an element.

Metrics with the same degree of homogeneity need not be equivalent. For example, for $n=3, \tau^{2}$ and $|T|^{6}$ are homogeneous of degree six, but are not equivalent.

Proposition 6.4

$f_{1} \sim f_{2}$ if and only if $\tilde{f}_{1} \sim \tilde{f}_{2}$.

\section{Proposition 6.5.}

Using singular values, $\kappa^{2}(T) \simeq \kappa\left(T^{t} T\right)$, since

$$
\frac{1}{3} \kappa^{2}(T) \leq \kappa\left(T^{t} T\right) \leq \kappa^{2}(T)
$$

$\S$

\section{Proposition 6.6}

Let $\kappa_{2}(T)=|T|_{2}\left|T^{-1}\right|_{2}$. Then $\kappa \sim \kappa_{2}$.

Proof.

Using the first line of the proof of Proposition 6.1, one can readily show that

$$
\kappa_{2} \leq \kappa \leq n \kappa_{2}
$$

$\S$

\section{Proposition 6.7}

For $n=3$, let $\mu(T)=\tau^{-2 / 3}|T|^{2}$ with conjugate $\mu^{*}(T)=\tau^{2 / 3}\left|T^{-1}\right|^{2}$. Then $\mu \sim \mu^{*} \sim \kappa$.

Proof

Let $0<\lambda_{1} \leq \lambda_{2} \leq \lambda_{3}$ be the eigenvalues of of $T^{t} T$.

Part A, $\mu \sim \kappa$.

$$
\mu=\frac{1+\lambda_{2} / \lambda_{1}+\lambda_{3} / \lambda_{1}}{\left[\left(\lambda_{2} / \lambda_{1}\right)\left(\lambda_{3} / \lambda_{1}\right)\right]^{1 / 3}}
$$


Therefore,

$$
\begin{array}{r}
\left(\lambda_{3} / \lambda_{1}\right)^{1 / 3} \leq \mu \leq 3\left(\lambda_{3} / \lambda_{1}\right)^{2 / 3} \\
\kappa_{2}^{1 / 3} \leq \mu \leq 3 \kappa_{2}^{2 / 3} \\
(\kappa / 3)^{1 / 3} \leq \mu \leq 3^{5 / 3}(\kappa / 3)^{2 / 3}
\end{array}
$$

Part $\mathrm{B}, \mu^{*} \sim \kappa$.

$$
\mu^{*}=\left[\left(\lambda_{2} / \lambda_{3}\right)\left(\lambda_{1} / \lambda_{3}\right)\right]^{1 / 3}\left(1+\lambda_{3} / \lambda_{2}+\lambda_{3} / \lambda_{1}\right)
$$

Therefore,

$$
\begin{array}{r}
\left(\lambda_{3} / \lambda_{1}\right)^{1 / 3} \leq \mu^{*} \leq 3 \lambda_{3} / \lambda_{1} \\
\kappa_{2}^{2 / 3} \leq \mu^{*} \leq 3 \kappa_{2}^{2} \\
(\kappa / 3)^{2 / 3} \leq \mu^{*} \leq 27(\kappa / 3)^{2}
\end{array}
$$

Then by the definition of equivalences, $\mu \sim \mu^{*}$. $\S$

For $n=2$, the corresponding scale-invariant metric is $\mu=|T|^{2} / \tau$. In this case it is easy to show that $\mu=\mu^{*}=\kappa$.

We began this section by giving the definition of a tetrahedral shape measure. The definition is vague on the definition of a degenerate element. In the next section we fix this and define algebraic shape metrics.

\section{Algebraic Shape Metrics and the Condition Number}

We formalize the definition of a degenerate element by first defining a degenerate matrix.

Definition Let $B \in \mathcal{M}_{n}^{+} \cup \partial \mathcal{M}_{n}^{+}$. Then $\mathrm{B}$ is degenerate if $\mathrm{B}$ is singular but non-zero (i.e., $|B|>0$ with $\operatorname{det}(B)=0$ ). $\mathrm{B}$ is non-degenerate if $\operatorname{det} B>0$, i.e., $B \in \mathcal{M}_{n}^{+}$. Let $\mathcal{D G}(n)$ be the set of degenerate $n \times n$ matrices. The set of singular matrices $\partial M_{n}^{+}$ then consists of $\mathcal{D G}(n)$ plus the zero matrix.

Definition A simplicial element $\varepsilon_{n}$ is degenerate if and only if the matrices $A_{k}$, $k=0,1, \ldots, K-1$, are degenerate. Sliver elements are "near-degenerate" elements.

\section{Proposition 7.1}

$\varepsilon_{n}$ is degenerate if and only if the matrix $T$ is degenerate.

Proof

If $\varepsilon_{n}$ is degenerate then $A_{k}$ is degenerate for all k. Since $T=A_{k} W_{k}^{-1}, \tau=\operatorname{det}(T)=$ $\alpha_{k} / w_{k}=0$. Hence $\mathrm{T}$ is singular. Suppose $T=\mathcal{O}$. Then $\mathcal{O}=A_{k} W_{k}^{-1}$, which gives $A_{k}=\mathcal{O}$ and $\left|A_{k}\right|=0$. But since $A_{k}$ is degenerate, its norm must be strictly positive. To avoid this contradiction we must have $|T|>0$, i.e., $T$ is degenerate. The proof in the other direction is similar. $\S$

As a reminder, we assume here and in subsequent sections that $\alpha \geq 0,0<|A|<$ $\infty$ and that $\mathrm{W}$ is non-degenerate. 
We return to the factorization of the Jacobian matrix discussed in section 3.1. As observed, the Jacobian matrix contains the following information, skew (Q), length ratio (D), shape (S), volume (U), and orientation (R). It should therefore be possible to define algebraic mesh quality metrics for each of these geometric quantities. In this section we will consider algebraic shape metrics. Let

$$
\begin{aligned}
A & =\mu R S \\
W & =\mu_{W} R_{W} S_{W}
\end{aligned}
$$

The shape of A will equal the shape of W if $S=S_{W}$. We adapt the Dompierre definition of shape measures to the algebraic setting. ${ }^{6}$

Definition.

Let $f$ be an algebraic mesh quality metric. Then $f$ is an algebraic Shape metric if

- the domain of $f$ is restricted to the matrix $T$,

- $f$ is scale and orientation invariant,

- $0 \leq f(T) \leq 1$, for all $T$,

- $f(T)=1$ if and only if $T \in \mathcal{S R}(n),{ }^{7}$

- $f(T)=0$ if and only if $T$ is degenerate.

\section{Proposition 7.2}

Algebraic shape metrics are invariant to uniform scalings and rotations of the physical element.

Proof

Uniform scalings and rotations of an element mean that $A_{k} \rightarrow B A_{k}$, where $B \in$ $\mathcal{S R}(n)$. Then $T=A_{k} W_{k}^{-1} \rightarrow B T$. But by definition, $f(B T)=f(T)$.

\section{Proposition 7.3}

$f(T)=n / \kappa(T)$ is an algebraic shape metric.

Proof

The first criterion is immediate. Secondly, because $T$ is nodally-invariant, $f$ is invariant to the node at which it is computed. Observe that

$$
\kappa^{2}(T)=\sum_{i} \sum_{j} \frac{\lambda_{i}}{\lambda_{j}}
$$

with $\lambda_{i}$ the eigenvalues of $T^{t} T$. Setting $\partial \kappa / \partial \lambda_{i}=0$ to find the extremum, one finds that $n \leq \kappa<\infty$, hence $0 \leq f \leq 1$. If $f=1$, then $\kappa=n$, i.e., $\lambda_{i}=\lambda_{j}$ for all $i, j$. Therefore, by the singular value decomposition, $T=\lambda_{i} \Theta$, i.e., $T \in \mathcal{S R}(n)$. If $T \in \mathcal{S R}(n)$, then $\kappa=n$, so $f=1$. This proves $f$ meets the third and fourth requirements. Fifth, if $\mathrm{T}$ is degenerate, then $\lambda_{1}=0$ and so $\kappa \rightarrow \infty$ and $f=0$. Finally, if $f=0$, then $\kappa \rightarrow \infty$, and so $\lambda_{1}=0$ and $\lambda_{3}>0$, so $\mathrm{T}$ is degenerate. $\S$

\footnotetext{
${ }^{6}$ In our definition we do not say anything about the metric lacking local minimae or maximae. The property is related to the convexity of $f$ with respect to $T$. This condition, while highly desirable, is probably too restrictive in most cases, i.e., if added to the definitions, there will be no function that can satisfy all of the requirements. Numerical results in section 11 show that the metrics we suggest do not possess local extremae with respect to some parameters, but perhaps not all.

7 This requirement forces $S=S_{W}$ when $f=1$.
} 
Similarly, one can prove $3 / \mu(T)$ and $3 / \mu^{*}(T)$ are algebraic shape metrics. ${ }^{8}$

The distinguishing property of the condition number is given in the following well-known theorem [5] adapted to our current setting.

Proposition 7.4.

$1 / \kappa_{2}(X)$ is the greatest lower bound for the distance of $X$ to the set of singular matrices.

Proof.

Let $X$ and $Y$ be $3 \times 3$ matrices with $X$ non-singular and $X+Y$ singular. Write $X+Y=X\left(I+X^{-1} Y\right)$. If $\left|X^{-1} Y\right|_{2}<1$, then $I+X^{-1} Y$ is nonsingular. This would mean that $X+Y$ is nonsingular, so we must have $\left|X^{-1} Y\right|_{2} \geq 1$. But $1 \leq\left|X^{-1} Y\right|_{2} \leq\left|X^{-1}\right|_{2}|Y|_{2}$; hence $|Y|_{2} /|X|_{2} \geq 1 / \kappa_{2}(X)$. Therefore

$$
d \equiv \min \left\{|Y|_{2} /|X|_{2}: X+Y \text { singular }\right\}=1 / \kappa_{2}(X) .
$$

$\S$

Proposition 7.5

$f=3 / \kappa$ is an equivalent measure of the minimum distance to the set of singular matrices.

Proof

From Proposition $6.6, \kappa_{2} \sim \kappa$, i.e.,

$$
\kappa_{2} \leq \kappa \leq 3 \kappa_{2}
$$

we have

$$
\frac{f}{3} \leq d \leq f
$$

i.e., d goes to zero if and only if $f$ goes to zero.

$\S$

Corollary.

Since $\mathcal{D G}$ is a subset of the singular matrices, $f$ also measures the distance to degenerate matrices and thus the distance to degenerate elements. $\S$

\section{Algebraic Metrics for Skew and Length Ratio}

The algebraic shape metrics, as defined in the previous section, are invariant to the node at which they are computed. Unfortunately, the elegant way in which this is achieved by using the matrix $\mathrm{T}$ cannot be done for properties such as skew and length ratio. To create nodally-invariant skew metrics, we can define functions that use matrices at all of the nodes. ${ }^{9}$

\section{Definition.}

Let $f$ be an algebraic mesh quality metric. Then $f$ is a algebraic Skew metric if

\footnotetext{
${ }^{8}$ Note that for $n=3$ the Winslow metric $\tau\left|T^{-1}\right|^{2}$ is not a shape metric because it is not scale-invariant. From the definition in section 10 , it is not a shape-volume metric either. This may explain why 3D winslow smoothing of structured grids has had only limited success.

${ }^{9}$ Shape metrics can also be defined in this way, using $X_{k}=S_{k} S_{W_{k}}^{-1}$. Then if $f$ is orientation invariant, $f\left(\left\{X_{k}\right\}\right)=f\left(\left\{\mu R X_{k} R_{W}^{-1} \mu_{W}^{-1}\right\}\right)=f\left(\left\{A_{k} W_{k}^{-1}\right\}\right)=f(T)$.
} 
- the domain of $f$ consists of the matrices $X_{k}=Q_{k} Q_{W_{k}}^{-1}, k=0,1, \ldots, K-1$, in the decompositions of $A_{k}$ and $W_{k}$,

- $0 \leq f\left(\left\{X_{k}\right\}\right) \leq 1$ for all matrices $X_{k}$,

- $f\left(\left\{X_{k}\right\}\right)=1$ if and only if $X_{k}=\mathcal{I}_{n}$ for all $\mathrm{k}$,

- $f\left(\left\{X_{k}\right\}\right)=0$ if and only if $X_{k}$ is degenerate for at least one $\mathrm{k}$.

\section{Proposition 8.1}

Algebraic skew metrics are invariant to uniform scalings and rotations of the physical element.

Proof

Under such an element transformation, $A_{k} \rightarrow B A_{k}$ where $B \in \mathcal{S R}(n)$. Then $Q_{k}=$ skew $A_{k} \rightarrow$ skew $B A_{k}=Q_{k}$. Thus $X_{k}$ is unchanged under element scaling and rotation. $\S$

\section{Proposition 8.2}

If $X_{k}=Q_{k} Q_{W_{k}}^{-1}$, then $f=\prod_{k} \frac{n}{\kappa\left(X_{k}\right)}$ is an algebraic skew metric.

Proof

By construction, $f$ is nodally-invariant because it uses all nodes, so $f$ is an algebraic metric. The remainder of the proof relies on the facts about $n / \kappa$ noted in Proposition 7.3. If $f=1$, then for all $\mathrm{k}, X_{k} \in \mathcal{S R}(n)$, i.e., $Q_{k}$ can differ from $Q_{W_{k}}$ only by a rotation and scaling. But since these two matrics are both skew matrices, we must have $Q_{k}=Q_{W_{k}}$, hence $X_{k}=\mathcal{I}_{n}$. If $f=0$ then $\operatorname{det}\left(X_{k}\right)=0$ for some k. Furthermore, $\sqrt{n}\left|X_{k}\right|=\left|X_{k}\right|\left|Q_{W_{k}}\right| \geq\left|X_{k} Q_{W_{k}}\right|=\left|Q_{k}\right|=\sqrt{n}>0$. Hence $\left|X_{k}\right|=1$ and $X_{k}$ is degenerate. $\S$

\section{Proposition 8.3}

$f=\min _{k}\left\{\frac{n}{\kappa\left(X_{k}\right)}\right\}$ is an algebraic skew metric. $\S$

\section{Definition.}

Let $f$ be an algebraic mesh quality metric. Then $f$ is an algebraic Length Ratio metric if

- The domain of $f$ consists of the matrices $X_{k}=D_{k} D_{W_{k}}^{-1}, k=0,1, \ldots, K-1$, in the decompositions of $A_{k}$ and $W_{k}$,

- $0 \leq f\left(\left\{X_{k}\right\}\right) \leq 1$ for all $X_{k}$,

- $f\left(\left\{X_{k}\right\}\right)=1$ if and only if $X_{k}=\mathcal{I}_{n}$ for all $\mathrm{k}$,

- $f\left(\left\{X_{k}\right\}\right)=0$ if and only if $X_{k}$ is degenerate for at least one node.

\section{Proposition 8.4}

Algebraic length ratio metrics are invariant to scalings and rotations of the element. $\S$

\section{Proposition 8.5}

By this definition, $f=\prod_{k} n / \kappa\left(X_{k}\right)$ and $f=\min _{k} n / \kappa\left(X_{k}\right)$ are algebraic length ratio metrics.

Proof

The proof is similar to proposition 8.2. $\S$

\section{Algebraic Metrics for Volume and Orientation}

For the definition of an orientation metric, let three diagonal matrices $\Psi^{\ell} \in \mathcal{S O}(3)$, 
$\ell=1,2,3$, be defined as follows

$$
\Psi_{i j}^{\ell}=\left\{\begin{array}{cc}
1 & i=j=\ell \\
-1 & i=j \neq \ell \\
0 & i \neq j
\end{array}\right.
$$

\section{Definition}

Let $f$ be an algebraic mesh quality metric. Then $f$ is an algebraic Orientation metric if

- The domain of $f$ is restricted to matrix $X_{0}=R_{0} R_{W_{0}}^{-1}$,

- $0 \leq f\left(X_{0}\right) \leq 1$ for all $X_{0}$,

- $f\left(X_{0}\right)=1$ if and only if $X_{0}=\mathcal{I}_{n}$,

- $f\left(X_{0}\right)=0$ if and only if $X_{0}=-\mathcal{I}_{n}$ when $n=2$, and $X_{0}=\Psi^{\ell}$ for some $\ell$ when $n=3$.

Algebraic orientation metrics are nodally-invariant because the nodes on which they depend are specified. However, they critically depend on the node numbering scheme of the element (i.e., which node is numbered zero).

\section{Proposition 9.1}

Algebraic orientation metrics are invariant to uniform scalings of the physical element. Proof

The proof is immediate since uniform scaling does not affect $R_{0}$ and thus $X_{0}$. $\S$

\section{Proposition 9.2}

$f\left(X_{0}\right)=1+\left(\operatorname{trace}\left(X_{0}\right)-n\right) / 4$ is an algebraic orientation metric. So is $f\left(X_{0}\right)=$ $1-\frac{1}{8}\left|X_{0}-\mathcal{I}_{n}\right|^{2}$.

\section{Proof}

Consider the first statement. Since $X_{0} \in \mathcal{S O}(n),\left|X_{i_{2}}\right| \leq 1$. Then we must have $n-4 \leq \operatorname{trace}\left(X_{0}\right) \leq n$, which gives $0 \leq f\left(X_{0}\right) \leq 1$. Suppose $f\left(X_{0}\right)=1$. Then trace $X_{0}=n$, which forces $X_{0}=\mathcal{I}_{n}$. Suppose trace $X_{0}=0$. Then trace $X_{0}=n-4$, which, for $n=2$, forces $X_{0}=-\mathcal{I}_{2}$ and, for $n=3$, forces $X_{0}=\Psi^{\ell}$. The proof of the second statement is similar. $\S$

The volume of an element depends both on edge lengths and element skew. A referenced volume metric is defined below.

\section{Definition.}

Let $f$ be an algebraic mesh quality metric. Then $f$ is an algebraic Volume metric if

- The domain of $f$ is restricted to the matrix $T$,

- $f$ is orientation invariant,

- $f$ is homogeneous of degree $\mathrm{n}$,

- $0 \leq f(T)<\infty$ for all $T$,

- $f(T)=1$ if and only if $T \in Z(n)$,

- $f(T)=0$ if and only if $T$ is degenerate.

A value of $f$ greater (less) than one means the physical element has volume greater (less) than the volume of the reference element. Since element volume is unbounded, the upper limit of $f$ is unbounded. 
Proposition 9.3

$f(T)=\operatorname{det}(T)$ is an algebraic mesh volume metric. $\S$

If $f(T)=1$ then $A_{k}=H W_{k}$ where $H \in \mathcal{Z}(n)$. Therefore the volume of the element is the same as the reference element, but the shape may differ.

\section{Combination Metrics}

Combinations of the various metrics are often more useful than single metrics. Below we define algebraic volume-shape metrics.

\section{Definition}

Let $f$ be an algebraic mesh quality metric. Then $f$ is an algebraic Volume-Shape metric if,

- The domain of $f$ is restricted to the matrix $T$,

- $f$ is orientation-invariant,

- $0 \leq f(T) \leq 1$ for all $T$,

- $f(T)=1$ if and only if $T \in \mathcal{S O}(n)$,

- $f(T)=0$ if and only if $T$ is degenerate.

Proposition 10.1

Algebraic volume-shape metrics are invariant to rotations of the physical element. $\S$

If the requirement that $f$ be homogeneous of degree $\mathrm{n}$ is included in the definition of a volume-shape metric, we cannot find specific examples. For example, adding the homogeneity requirement in one attempt resulted in a discontinuous function - not allowed under the definition of an algebraic metric.

\section{Proposition 10.2}

Define

$$
f(T)=\min (\tau, 1 / \tau) n / \kappa(T)
$$

Then $f(T)$ is an algebraic volume-shape metric.

Proof.

$f$ is continuous because $\lim _{\tau \rightarrow 1} f$ is the same whether one approaches from above or below. Suppose $\tau \leq 1$. Since $\operatorname{det}(T) \leq 1$ and $\operatorname{det}(T) n / \kappa(T) \leq 1$ for any $T, f(T)=1$ forces $\operatorname{det}(T)=1$ and $n / \kappa(T)=1$. Thus $T \in \mathcal{Z}_{n} \cap \mathcal{S R}(n)=\mathcal{S O}(n)$. Similarly, if $\operatorname{det}(T)>1, T \in \mathcal{S O}(n)$. If $f=0$, then $\tau \leq 1$ and either $\operatorname{det}(T)=0$ or $n / \kappa(T)=0$. Since $|T|>0, \mathrm{~T}$ is degenerate. $\S$

$f$ in the previous Proposition is homogeneous of degree $\mathrm{n}$ when $\tau<1$ and homogeneous of degree $-n$ when $\tau>1$.

It is possible in a similar manner to define and give examples of combined shapeorientation and volume-orientation metrics. More useful, however, is the following volume-shape-orientation metric.

\section{Definition}

Let $f$ be an algebraic mesh quality metric. Then $f$ is an algebraic Volume-ShapeOrientation metric if, 
- The domain of $f$ is restricted to the matrices $T$ and $X_{0}=R_{0} R_{W_{0}}^{-1}$,

- $0 \leq f\left(T, X_{0}\right) \leq 1$ for all $T$ and $X_{0}$,

- $f\left(T, X_{0}\right)=1$ if and only if $T=\mathcal{I}_{n}=X_{0}$

- $f(T)=0$ if and only if $T$ is degenerate or $X_{0}=-\mathcal{I}_{n}$ for $n=2$ and $X_{0}=\Psi^{\ell}$ for $\ell=1,2$ or 3 .

\section{Proposition 10.3}

Let $g(T)=n / \kappa(T), h\left(X_{0}\right)=1+\frac{1}{4}\left(\operatorname{trace}\left(X_{0}\right)-n\right)$, and

$$
f\left(T, X_{0}\right)=\min (\tau, 1 / \tau) g(T) h\left(X_{0}\right)
$$

Then $f\left(T, X_{0}\right)$ is an algebraic volume-shape-orientation metric.

Proof.

Suppose $\tau \leq 1$. Since $\operatorname{det}(T) \leq 1, n / \kappa(T) \leq 1$, and $h\left(X_{0}\right) \leq 1$ for any $T, f(T)=1$ forces $\operatorname{det}(T)=1, n / \kappa(T)=1$, and $h\left(X_{0}\right)=1$. Thus $T \in \mathcal{Z}_{n} \cap \mathcal{S R}(n) \cap \mathcal{I}_{n}=\mathcal{I}_{n}$. Similarly, if $\operatorname{det}(T)>1, T=\mathcal{I}_{n}$. If $f=0$, then either $\operatorname{det}(T)=0, n / \kappa(T)=0$ or $h\left(X_{0}\right)=0$. Since $|T|>0, \mathrm{~T}$ is degenerate and $X_{0}=\mathcal{I}_{n}$ for $n=2$ and $X_{0}=\Psi^{\ell}$ for $\ell=1,2$ or 3 . $\S$

\section{Numerical Examples}

We have given general definitions of algebraic metrics for simplicial elements including shape, skew, length ratio, volume, orientation, and combinations thereof. Using the specific examples given in sections $7,8,9$. and 10 , we illustrate the behavior of these metrics with several test cases.

In the first test (see Figure 1) the metrics are ploted vs. the included angle of a triangular physical element with sides of unit length emanating from the origin. The first side lies on the $x$-axis, while the second side is oriented by a variable included angle. The reference triangle is the unit equilateral triangle with base on the $\mathrm{x}$-axis. Figure 1 shows that all the metrics except volume vary between zero and unity, as desired. Shape and length ratio peak when the included angle matches the sixtydegree angle of the reference triangle. The skew curve is not plotted, because it is nearly identical to the shape plot (because the relative lengths of sides of the physical triangle are the same as the reference triangle). The volume metric (p1-size) peaks at 1.15 when the included angle is ninety-degrees, i.e., the area of the physical triangle is 1.15 times the area of the reference triangle. The orientation of the physical triangle was varied by an angle from the $x$-axis. The results for the orientation metric in Figure 1 show a cosine curve, which agrees with theory. The combined shape and volume metric ( $\mathrm{p} 1-\mathrm{ss})$ is similar to the shape metric, but less smooth and with lower values.

In the second test (Figure 2) the same physical triangle was used except that the length of the second side was increased to 2, the base of the triangle made an angle of 30-degrees with the $\mathrm{x}$-axis, and the reference triangle was an isoceles triangle (perhaps describing some desired anisotropy in the mesh), with base 1 and height 2, As the included angle was varied from zero to 180 degrees, the angle shown in the plots varied from 30 to 210-degrees. The metrics ranged between zero and unity, peaking around 105-degrees for shape and 120-degrees for volume. The skew curve again overlaid the shape curve.

In the third test (Figure 3), the physical triangle had a unit length base which made an angle of 30-degrees with the $\mathrm{x}$-axis. The included angle between the first 
and second sides was 75-degrees. The length of the second side was varied from zero to three. The reference triangle was the same as in the second test. The shape and skew curves differed from each other somewhat because of the differences in lengths between the physical and reference triangles. In general, however, shape, skew, and length ratio followed the same trend as one another, peaking when the second length matched the reference triangle. Volume varied linearly with the variation in the length of the second side, as expected.

In our opinion, shape, volume, and combined shape-volume are the most valuable of the metrics. Skew varies nearly the same as shape while length ratio is misleading because it is not the ratio of element width to breadth but rather the ratio of the lengths of consecutive sides. Orientation may be of use provided element nodes can be numbered in a consistent manner.

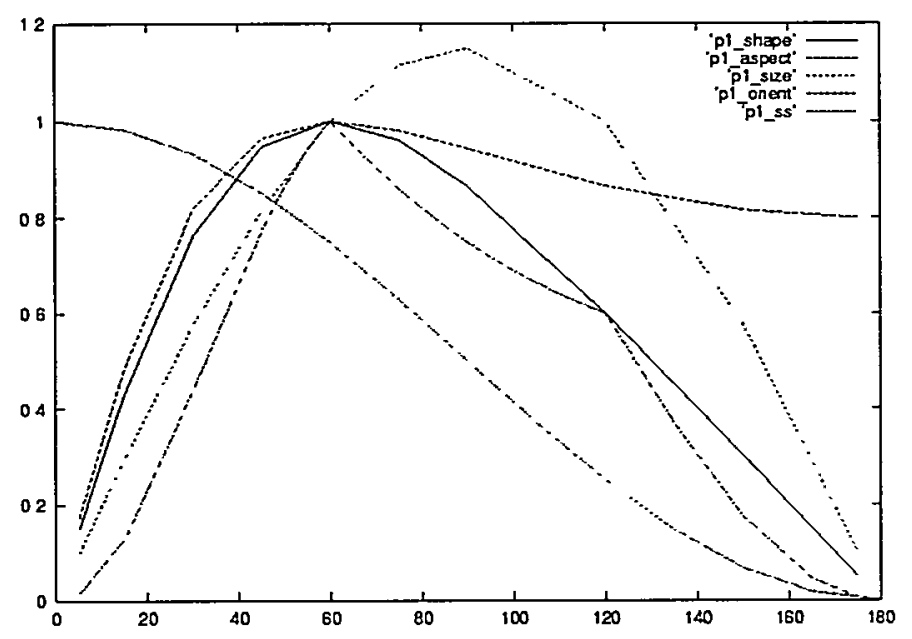

FIG. 1. Unit equilateral reference triangle, included angle varied

\section{Non-simplicial Element Metrics}

Non-simplicial elements such as quadrilaterals, hexahedra, and wedges fail to obey Propositions 3.5, 3.6, and 3.7. ${ }^{10}$ There is no single nodally-invariant matrix $\mathrm{T}$ which can represent all the geometric properties of non-simplicial elements. To build algebraic quality metrics for such elements we can resort to the technique used in section 8 , in which multiple matrices are used in the definition of the metric. Nonsimplicial elements for which Jacobian matrices $A_{k}$ can be defined may be treated as follows. Choose a reference element and compute the reference weight matrices $W_{k}$. Let $T_{k}=A_{k} W_{k}^{-1}, k=0,1, \ldots, K-1$ be the weighted matrix, where $\mathrm{K}$ is the number of nodes in the element. The matrices are factored as $A_{k}=R_{k} U_{k}=R_{k} Q_{k} \Delta_{k}$ and similarly for $W_{k}$. Basic assumptions are that $\alpha_{k} \geq 0,0<\left|A_{k}\right|<\infty$ and that $W_{k}$ is

\footnotetext{
${ }^{10}$ Pyramids and other 3D element having more than three edges meeting in a node are still more problematic since the Jacobian matrix fails to exist.
} 


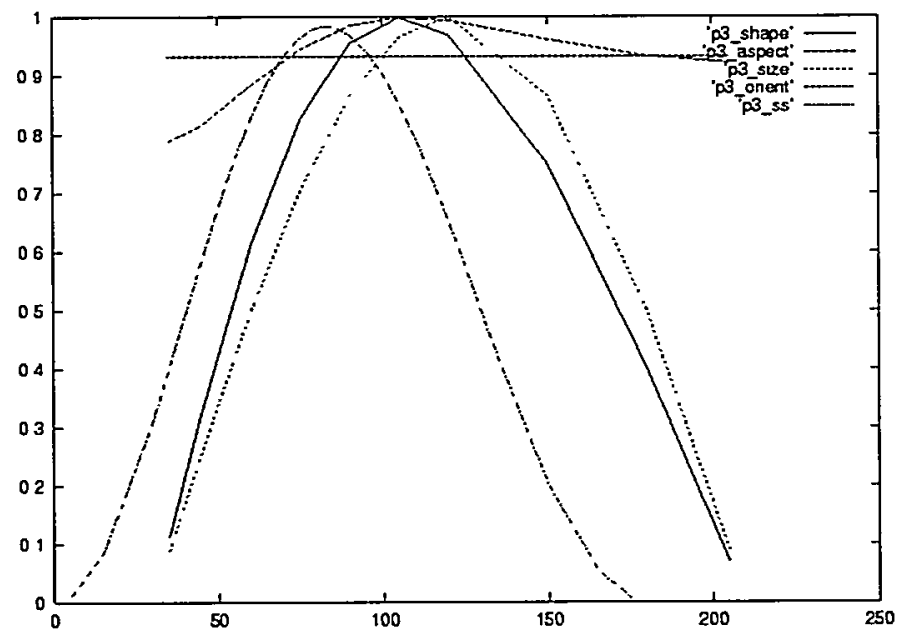

FIG. 2. Isoceles reference triangle, included angle varied

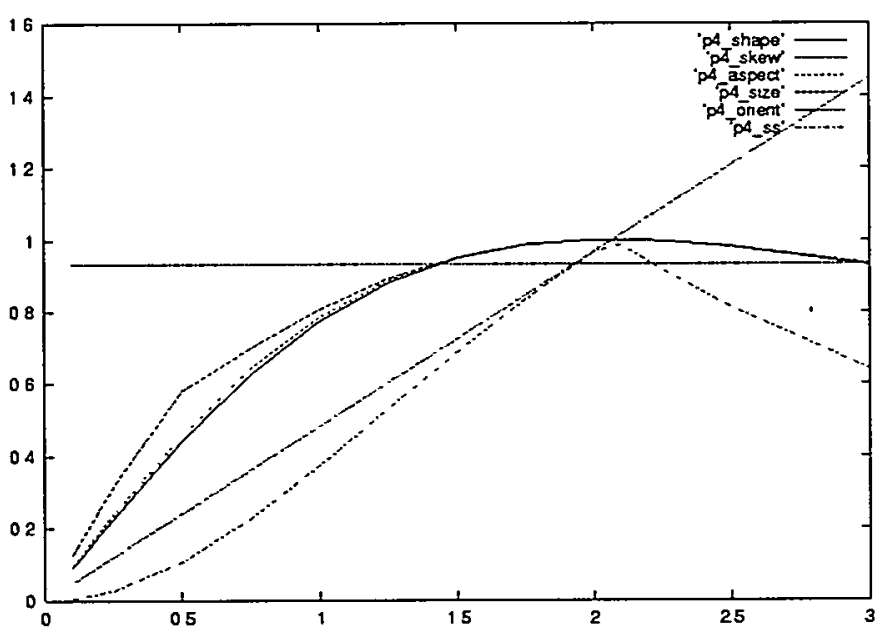

FIG. 3. Isoceles reference triangle, second side length varied

non-degenerate for all $\mathrm{k}$. The $W_{k}$ should be self-consistent, i.e., computed from an element that exists.

Shape and volume metrics are defined for non-simplicial elements, with the others left to the reader.

\section{Definition.}


Let $f$ be an algebraic mesh quality metric. Then $f$ is a non-simplicial algebraic shape metric if

- The domain of $f$ is the complete set of matrices $T_{k}=A_{k} W_{k}^{-1}, k=0,1, \ldots, K-$ 1 ,

- $f$ is scale and orientation invariant,

- $0 \leq f\left(\left\{T_{k}\right\}\right) \leq 1$, for all $T_{k}$,

- $f\left(\left\{T_{k}\right\}\right)=1$ if and only if $T_{k} \in \mathcal{S O}(n)$ for all $\mathrm{k}$,

- $f\left(\left\{T_{k}\right\}\right)=0$ if and only if $T_{k}$ is degenerate for some $\mathrm{k}$.

Proposition 11.1

$f\left(\left\{T_{k}\right\}\right)=\min _{k}\left\{n / \kappa\left(T_{k}\right)\right\}$ is an algebraic shape metric for non-simplicial elements. $\S$

If the definition of a volume metric given for simplicial elements in section 9 is directly extended to non-simplicial elements, the metric

$$
f\left(\left\{T_{k}\right\}\right)=\min _{k}\left\{\operatorname{det}\left(T_{k}\right)\right\}
$$

fails to satisfy the requirements because $f=1$ does not force $T_{k} \in \mathcal{Z}(n)$ for all $\mathrm{k}$. Other attempts to fix this also fail. We thus re-define algebraic volume metrics as follows:

\section{Definition}

Let $f$ be an algebraic mesh quality metric. The $f$ is an algebraic volume metric if

- The domain of $f$ is restricted to the matrices $T_{k}, k=0,1,2, \ldots, K-1$,

- $f$ is orientation invariant,

- $0 \leq f\left(\left\{T_{k}\right\}\right) \leq 1$ for all $T_{k}$,

- $f\left(\left\{T_{k}\right\}\right)=1$ if and only if $T_{k} \in Z(n)$ for all $k$,

- $f\left(\left\{T_{k}\right\}\right)=0$ if and only if $T_{k}$ is degenerate for some $\mathrm{k}$.

Proposition 11.2

$f\left(\left\{T_{k}\right\}\right)=\min _{k}\left\{\min \left(\tau_{k}, 1 / \tau_{k}\right)\right\}$ is an algebraic volume metric for non-simplicial elements. $\S$

If the value of the volume metric is say, $1 / 2$, then either the physical element has half or twice the volume of the reference element.

The definition of volume-shape metrics given in section 10 readily extends to the non-simplicial case.

Proposition 11.2

$f\left(\left\{T_{k}\right\}\right)=\min _{k}\left\{\min \left(\tau_{k}, 1 / \tau_{k}\right) n / \kappa\left(T_{k}\right)\right\}$ is an algebraic volume-shape metric for nonsimplicial elements. $\S$

Figure Four shows how such metrics vary for a quadrilateral element referenced to a unit square. The quadrilateral is a symmetric trapezoid, with a unit length base oriented in agreement with the reference element. The angle of the two vertical sides with respect to the base side was varied from 60 to 165 -degrees.

\section{Summary and Conclusions.}

A theory of algebraic mesh quality metrics was proposed based on element Jacobian matrices. Jacobian matrices can be decomposed into geometrically meaningful 


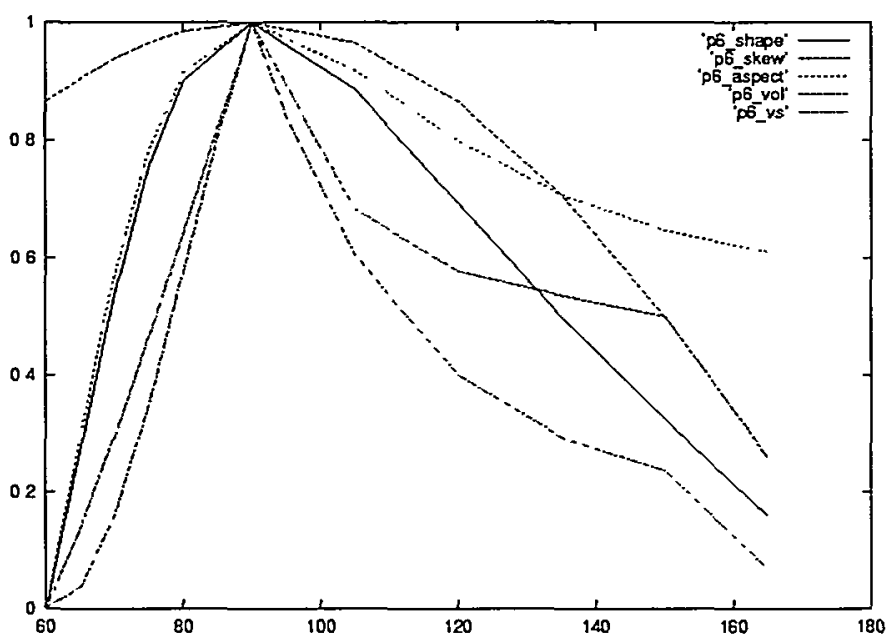

FIG. 4. Square reference quadrilateral, trapezoid physical element

factors representing element volume, orientation, and shape. The factor matrices are node-dependent and thus cannot be used to construct algebraic mesh quality measures unless all are used in a symmetric way. However, for simplicial elements one can define a single nodally-invariant matrix $\mathrm{T}$ using the Jacobian matrices $W_{k}$ of a reference element. We emphasize the point that mesh quality metrics should be explicitly referenced to a logical element. Thus, for example, shape metrics may be referenced to an isoceles, equilateral, or right angled simplicial element, depending on the application. We list the properties which much be satisfied by an algebraic mesh quality metric. An algebraic definition of mesh quality metrics permits relatively easy analysis of the properties of a metric, for example, in terms of its singular values. Abstract definitions of metrics are given in terms of precise requirements for algebraic shape, length ratio, skew, volume, orientation, volume-shape, and volume-shape-orientation metrics. The abstract definitions are slightly subjective, especially in the range and domain of the metrics, but are largely non-controversial. The requirements in the abstract definitions clearly must be satisified by any algebraic metric purporting to be of a particular type. Specific examples for each type of metric are given. The examples, for the most part, are conspicuous in that they are new. Few traditional metrics (even were they referenced) will qualify under the definitions given, with the notable exceptions of mean ratio and determinant. Shape, volume, volume-shape metrics for simplicial elements can be posed in terms of the nodally invariant matrix $T$ while the other metrics must use a set of nodally-dependent matrices. Examples of volume-shape metrics are difficult to construct due to the large number of requirements they must satisfy. Volume-shape metrics are critical to adaptive meshing and it is significant that a rigorous definition and example has been provided. Except for volume, the metrics are scaled between zero and unity for ease of comparison. Multiple Jacobian matrices are needed in the definitions of metrics for non-simplicial elements due to the lack of an analogy to the matrix $T$. The rigorous definitions given 
for the various types of metrics have made it clear that it is not, in general, easy to devise metrics having all the right properties; this is especially true for non-simplicial element metrics. For example, to obtain the proper behavior for a volume metric for non-simplicial elements, we sacrificed the homogeneity requirement. The difficulties encountered suggest that one reason why so many mesh quality metrics have been defined in the past is that few metrics satisfy all of the requirements. Although the metric definitions given require metrics to satisfy rigorous criteria to qualify being a metric of a particular type, there remains some freedom to define alternative metrics. Redundant metrics can be eliminated by investigating possible equivalances via singular values. It was shown that the algebraic shape metric, condition number, measures the distance to the set of degenerate elements. Not all geometric properties of potential interest can be given in terms of an algebraic metric. For example, non-algebraic metrics based on solid angle or length-to-width ratios, cannot be expressed as algebraic metrics. However, there seems little need for these additional metrics since, for example, solid-angle-based shape metrics are equivalent to the algebraically-based mean ratio shape metric.

Future work may include extending the theory to higher-order finite elements having mid-side nodes as a means to measuring element curvature. Development of connections between algebraic element quality metrics and effects upon analysis error, efficiency, and robustness should be pursued. Finally, the metrics given are likely candidates for objective functions in mesh smoothing and optimization techniques.

\section{Acknowledgements}

The author wishes to thank Fred Howes, David Womble, Robert Leland, and Tim Tautges for their support.

\section{REFERENCES}

[1] Tinico-Ruiz and Barrera-Sanchez, Area Functionals in Plane Grid Generation, pp293-302, Proceedings of the 6th Intl Conference on Numerical Grid Generation in Computational Field Simulations, M Cross ed, Greenwhich, UK July 1998.

[2] J. Brackbill, J. Saltzman, Adaptive zoning for singular problems in two dimensions, J. Comp. Phys., 46, pp342-368, 1982.

[3] S. Canann, J. Tristano, M. Staten, An Approach to Combined Laplacian and OptimizationBased Smoothing for Triangular, Quadrilateral, and Quad-Dominant Meshes, Procedings of the 7th International Meshing RoundTable, Detroit, 1998.

[4] C.L. Chen, K.Y. Szema, and S.R. Chakravarthy, Optimization of Unstructured Grid, AIA.A 95-0217, 33rd Aerospace Sciences Meeting, January 1995, Reno NV.

[5] Demmel, J. Applied Numerical Linear Algebra, SIAM, Philadelphia, 1997.

[6] J. Dompierre, P. Labbe, F. Guibault, and R. Camerero, Proposal of Benchmarks for 3D Unstructured Tetrahedral Mesh Optimization, pages 459-478, Proceedings of the 7-th International Meshing RoundTable '98, Dearborn MI, 1998.

[7] Freitag, L. and Knupp, P. Tetrahedral Element Shape Optimization via the Jacobian Determinant and Condition Number, pp247-258, Proceedings of the 8th International Meshing RoundTable, Lake Tahoe CA, 1999.

[8] George, P.L. and Borouchaki, H. Delaunay Triangulation and Meshing, Hermes, Paris, 1998.

[9] Golub, G. and Van Loan, C., Matrix Computations, Johns Hopkins University Press, Baltimore, 1983.

[10] Ives, D. Geometric Grid Generation, In Surface Modeling, Grid Generation, and Related Issues in Computational Fluid Dynamic (CFD) Solutions, NASA CP-3291, 1995.

[11] O.P. Jacquotte, J. Cabello, A variational method for the optimization and adaption of grids in computational fluid dynamics, in Numerical Grid Generation in Computational Fluid Mechanics '88, S. Sengupta, et. al., eds., pp405-413, PineRidge Press, Swansea UK, 1988.

[12] Knupp, P. and Robidoux, N. A Framework for Variational Grid Generation: Conditioning the Jacobian Matrix with Matrix Norms, SIAM J. Sci. Comput., 2000. 
[13] Knupp, P. Matrix Norms and the Condition Number, pp13-22, Proceedings of the 8th International Meshing RoundTable, Lake Tahoe CA, 1999.

[14] Knupp, P. Achieving Finite Element Mesh Quality via Optimization of the Jacobian Matrix Norm and Associated Quantities, Part I - A Framework for Surface Mesh Optimization, to appear in Intl. J. Num. Meth. Engr., 2000

[15] Knupp, P. Achieving Finite Element Mesh Quality via Optimization of the Jacobian Matrix Norm and Associated Quantities, Part II - A Framework for Volume Mesh Optimization, to appear in Intl. J. Num. Meth. Engr., 2000

[16] G. Liao, Variational Approach to Grid Generation, Num. PDE's, 8, pp143-147, 1992.

[17] Liu, A. and B. Joe, Relationship between tetrahedron quality measures, Bit, 34:268-287, 1994.

[18] Oddy, A., Goldak, J., McDill, M., Bibby, M., A distortion metric for isoparametric elements, Trans. CSME, No. 38-CSME-32, Accession No. 2161, 1988.

[19] V.N. Parthasarathy, C.M. Graichen, and A.F. Hathaway, A comparison of tetrahedron qualilty measures, Finite Elements in Analysis and Design, Vol. 15, pp255-261, 1993.

[20] Robinson, J. and Haggenmacher, G., Element warning diagnostics, Finite Element News, June-August, 1982.

[21] Robinson, J., Some new distortion measures for quadrilaterals, Finite Element Analysis, Vol. 3, p183-197, 1987.

[22] J. Robinson, CRE method of element testing and the Jacobian shape parameters, Eng. Comput., Vol. 4, pages 113-118, June 1987.

Sandia is a multuprogram laboratory operated by Sandia Corpmration, a Lockheed Martin Company. For the United States Deparment of Energy under contract DE-A(04-9+AL\$5000. 